\title{
Projective Curvature Tensor on $N(\kappa)$-Contact Metric Manifold Admitting Semi-Symmetric Non-Metric Connection
}

\author{
Mustafa Altın \\ Technical Sciences Vocational School Bingol University, Bingol, Turkey
}

\section{Article Info}

Keywords: $N(\kappa)$-contact metric manifolds, Projective curvature tensor, Semisymmetric non-metric connection 2010 AMS: 53C25, 53C35, 53D10

Received: 06 May 2020

Accepted: 18 August 2020

Available online: 15 December 2020

\begin{abstract}
The object of the present paper is to classify $N(\kappa)$-contact metric manifolds admitting the semi-symmetric non-metric connection with certain curvature conditions the projectively curvature tensor. We studied projective flat, $\xi$-projectively flat, $\phi$-projectively flat $N(\kappa)$ contact metric manifolds admitting the semi-symmetric non-metric connection. Also, we examine such manifolds under some local symmetry conditions related to projective curvature tensor.
\end{abstract}

\section{Introduction}

An almost contact metric manifold is a $(2 n+1)$-dimensional differentiable manifold with a structure $(\phi, \xi, \eta, g)$ such as

$$
\phi^{2}\left(W_{1}\right)=-W_{1}+\eta\left(W_{1}\right) \xi, \eta(\xi)=1, \quad \phi(\xi)=0, \quad \eta\left(\phi\left(W_{1}\right)\right)=0, g\left(\phi\left(W_{1}\right), \phi\left(W_{2}\right)\right)=g\left(W_{1}, W_{2}\right)-\eta\left(\left(W_{1}\right)\right) \eta\left(\left(W_{2}\right)\right)
$$

for any vector fields $W_{1}, W_{2} \in \chi(M)$, where $g$ is Riemannian metric, $\phi$ is a $(1,1)$-tensor field, $\xi$ is a vector field and $\eta$ is a $1-$ form on $M$ [1]. Blair, et al. [2] introduced the $(\kappa, \mu)$-nullity distribution of an almost contact metric manifold $M$ that is defined by

$$
\begin{aligned}
& N(\kappa, \mu): p \longrightarrow N_{p}(\kappa, \mu) \\
& N_{p}(\kappa, \mu)=\left\{W_{3} \in \Gamma\left(T_{p} M\right): R\left(W_{1}, W_{2}\right) W_{3}=(\kappa I+\mu h)\left[g\left(W_{2}, W_{3}\right) W_{1}-g\left(W_{1}, W_{3}\right) W_{2}\right]\right\}
\end{aligned}
$$

for all $W_{1}, W_{2} \in \Gamma(T M)$, where $\kappa$ and $\mu$ are real constants and $p \in M$. If $\xi \in N(\kappa, \mu)$, then $M$ is called $(\kappa, \mu)-$ contact metric manifold. If $\mu=0$, the $(\kappa, \mu)$-nullity distribution reduces to $\kappa$-nullity distribution.

The idea of $\kappa$-nullity distribution on a contact metric manifold was firstly presented by Tanno in 1988 [3]. $\kappa$-nullity distribution of an almost contact metric manifold $(M, \phi, \xi, \eta, g)$ is a distribution defined as

$$
N(\kappa): p \longrightarrow N_{p}(\kappa)=\left\{W_{3} \in \Gamma\left(T_{p} M\right): R\left(W_{1}, W_{2}\right) W_{3}=\kappa\left[g\left(W_{2}, W_{3}\right) W_{1}-g\left(W_{1}, W_{3}\right) W_{2}\right]\right\}
$$

for any $W_{1}, W_{2} \in \Gamma\left(T_{p} M\right)$ and $\kappa \in \mathbb{R}$, where $R$ is the Riemannian curvature tensor of $M$. If $\xi$ belongs to $\kappa-$ nullity distribution then $M$ is called $N(\kappa)$-contact metric manifold. Thus on a $N(\kappa)$ contact metric manifold, we have

$$
R\left(W_{1}, W_{2}\right) \xi=\kappa\left[\eta\left(W_{2}\right) W_{1}-\eta\left(W_{1}\right) W_{2}\right]
$$


A $N(\kappa)$-contact metric manifold is Sasakian if and only if $k=1$. Also, if $k=0$, then the manifold is locally isometric to the product $E^{n+1}(0) \times S^{n}(4)$ for $n>1$ and flat for $n=1$ [4]. The Riemannian geometry of $N(\kappa)-$ contact metric manifolds is studied in [2], [5]-[9]. Levi-Civita connection $\nabla$ is a torsion free, i.e has zero torsion, and a metric connection, i.e $\nabla g=0$. There are some kinds of linear connections except for Levi-Civita connection which is not need to be torsion free or metric. One of them is semi-symmetric non-metric connection [10]. Manifolds with semi-symmetric non-metric connection have been studied by many researchers [11]-[15]. In the Riemannian geometry of contact manifolds curvature tensors-such as conformal, concircular, projective curvature tensor etc.-have important applications. Some of geometric properties of structure on manifolds have been examined by the certain conditions on these curvature tensors. Many works on contact manifolds are stated in [16]-[23].

In this paper we study projective curvature tensor on $N(\kappa)$-contact metric manifolds with semi-symmetric non metric connection. In [24], Barman gave the curvature relations on such as manifolds. We use these properties and we examine flatness conditions of projective curvature tensor. Specifically, we given results for $\xi$-projectively flat, pseudo-quasi-projectively flat and $\phi$-projectively flat on $N(\kappa)$-contact metric manifolds with semi-symmetric non metric connection. After we investigate $\phi$-projectively semi-symmetric on $N(\kappa)$-contact manifolds admitting the semi-symmetric non-metric connection, we characterize this manifolds satisfying $\stackrel{\star}{Q} . \stackrel{\star}{P}=0$ and $\stackrel{\star}{S} . \stackrel{\star}{P}=0$, where $\stackrel{\star}{P}, \stackrel{\star}{Q}, \stackrel{\star}{\text { ic }}$ are projective curvature tensor, Ricci tensor, Ricci curvature tensor, with a semi-symmetric non metric connection, respectively.

\section{Preliminaries}

Let $(M, \phi, \xi, \eta, g)$ be an almost contact metric manifold. The $h=\frac{1}{2} \mathscr{L}_{\xi} \phi, \mathscr{L}_{\xi}$ denotes the Lie derivative along vector field $\xi$. For any $W_{1} \in \Gamma(T M)$, we have

$$
\nabla_{W_{1}} \xi=-\phi W_{1}-\phi h W_{1}
$$

An almost contact metric manifold $M$ is called $K$-contact if $\xi$ is Killing vector field. $M$ is called normal contact metric manifold if $N_{\phi}+2 d \eta \otimes \xi=0$, where $N_{\phi}$ is the Nijenhuis tensor of $\phi$. A normal contact metric manifold is called Sasakian. On the other hand a contact metric manifold is Sasakian if and only if

$$
R\left(W_{1}, W_{2}\right) \xi=\left[\eta\left(W_{2}\right) W_{1}-\eta\left(W_{1}\right) W_{2}\right]
$$

for all $W_{1}, W_{2} \in \Gamma(T M)$. On a $K$-contact and Sasakian manifold $h=0$.

A $N(\kappa)$-contact metric manifold is Sasakian if $\kappa=1 . N(\kappa)$-contact metric manifolds are characterized the different values of $\kappa$. As we mentioned in the introduction when $\kappa=0$ then the manifold $M$ is locally isometric to $\left.E^{(n+1}\right)(0) \times S^{(n)}(4)$. On a $N(\kappa)$-contact metric manifold $M^{2 n+1}$, we have following relations (for details see [1] ):

$$
\begin{aligned}
& \left(\nabla_{W_{1}} \phi\right) W_{2}=g\left(W_{1}+h W_{1}, W_{2}\right) \xi-\eta\left(W_{2}\right)\left(W_{1}+h W_{1}\right) \\
& \left(\nabla_{W_{1}} \eta\right) W_{2}=g\left(W_{1}+h W_{1}, \phi W_{2}\right) .
\end{aligned}
$$

The Riemannian curvature $R$ of a $N(\kappa)$-contact metric manifold has following properties:

$$
\begin{aligned}
& R\left(W_{1}, W_{2}\right) \xi=\kappa\left[\eta\left(W_{2}\right) W_{1}-\eta\left(W_{1}\right) W_{2}\right] \\
& R\left(\xi, W_{1}\right) W_{2}=\kappa\left[g\left(W_{1}, W_{2}\right) \xi-\eta\left(W_{2}\right) W_{1}\right]
\end{aligned}
$$

for all $W_{1}, W_{2} \in \Gamma(T M)$. On the other hand the Ricci curvature of $M$ is stated as [1];

$$
\begin{aligned}
\operatorname{Ric}\left(W_{1}, W_{2}\right) & =2(n-1) g\left(W_{1}, W_{2}\right)+2(n-1) g\left(h W_{1}, W_{2}\right)+2(n \kappa-(n-1)) \eta\left(W_{1}\right) \eta\left(W_{2}\right) \\
\operatorname{Ric}\left(\phi W_{1}, \phi W_{2}\right) & =\operatorname{Ric}\left(W_{1}, W_{2}\right)-2 n \kappa \eta\left(W_{1}\right) \eta\left(W_{2}\right)-4(n-1) g\left(h W_{1}, W_{2}\right) \\
\operatorname{Ric}\left(W_{1}, \xi\right) & =2 \kappa n \eta\left(W_{1}\right)
\end{aligned}
$$

and the scalar curvature is given by

$$
r=2 n(2 n+\kappa-2)
$$

Example 2.1. E. Boeckx [25] gave a classification for non-Sasakian $(\kappa, \mu)$-spaces. The number $I_{M}=\frac{1-\frac{\mu}{2}}{\sqrt{1-k}}$ is called by Boeckx invariant. D.E. Blair, et al. [26] gave an example of $N(\kappa)$-contact metric manifolds by using Boeckx invariant. They constructed $(2 n+1)$-dimensional $N\left(1-\frac{1}{n}\right)$-contact metric manifold, $n>1$. For details see [26].

Let define a map $\stackrel{\star}{\nabla}$ on a Riemann manifold $M$ as

$$
\stackrel{\star}{\nabla}_{W_{1}} W_{2}=\nabla_{W_{1}} W_{2}+\eta\left(W_{2}\right) W_{1}
$$


where $\nabla$ is Levi-Civita connection on $M$. This map is a linear connection. The torsion of $\stackrel{\star}{\nabla}$ is given by

$$
\stackrel{\star}{T}\left(W_{1}, W_{2}\right)=\eta\left(W_{2}\right) W_{1}-\eta\left(W_{1}\right) W_{2}
$$

for all $W_{1}, W_{2} \in \Gamma(T M)$. Also we have

$$
\left(\stackrel{\star}{\nabla}_{U} g\right)\left(W_{1}, W_{2}\right)=-\eta\left(W_{1}\right) g\left(W_{2}, U\right)-\eta\left(W_{2}\right) g\left(W_{1}, U\right) \neq 0
$$

Thus $\stackrel{\star}{\nabla}$ is not symmetric and not metric connection. This type of connection is called by semi-symmetric non-metric connection [10].

$N(\kappa)$ - contact metric manifolds with a semi-symmetric non-metric connection were studied by Barman [24]. For the sake of brevity we denote $(M, \stackrel{\star}{\nabla})$ by a $N(\kappa)$-contact metric manifolds with a semi-symmetric non-metric connection. Barman gave the curvature of $(M, \stackrel{\star}{\nabla})$ as follow:

$$
\begin{aligned}
\stackrel{\star}{R}\left(W_{1}, W_{2}\right) W_{3} & =R\left(W_{1}, W_{2}\right) W_{3}+g\left(W_{1}, \phi W_{3}\right) W_{2}+g\left(h W_{1}, \phi W_{3}\right) W_{2}-\eta\left(W_{1}\right) \eta\left(W_{3}\right) W_{2}-g\left(W_{2}, \phi W_{3}\right) W_{1} \\
& -g\left(h W_{2}, \phi W_{3}\right) W_{1}+\eta\left(W_{3}\right) \eta\left(W_{2}\right) W_{1}
\end{aligned}
$$

Thus, we have following curvature properties [24]:

$$
\begin{aligned}
& \stackrel{\star}{R}\left(\xi, W_{2}\right) W_{3}=\kappa g\left(W_{2}, W_{3}\right) \xi-(\kappa+1) \eta\left(W_{3}\right) W_{2}-g\left(W_{2}, \phi W_{3}\right) \xi-g\left(h W_{2}, \phi W_{3}\right) \xi+\eta\left(W_{3}\right) \eta\left(W_{2}\right) \xi \\
& \stackrel{\star}{R}\left(\xi, W_{2}\right) \xi=(\kappa+1)\left(\eta\left(W_{2}\right) \xi-W_{2}\right) \\
& \stackrel{\star}{R}\left(W_{1}, W_{2}\right) \xi=(\kappa+1)\left(\eta\left(W_{2}\right) W_{1}-\eta\left(W_{1}\right) W_{2}\right) .
\end{aligned}
$$

The Ricci curvature of a $(M, \stackrel{\star}{\nabla})$ is given by

$$
\stackrel{\star}{\operatorname{Ric}}\left(W_{2}, W_{3}\right)=\operatorname{Ric}\left(W_{2}, W_{3}\right)-2 n g\left(W_{2}, \phi W_{3}\right)-2 n g\left(h W_{2}, \phi W_{3}\right)+2 n \eta\left(W_{3}\right) \eta\left(W_{2}\right) .
$$

Thus, we have

$$
\begin{aligned}
\stackrel{\star}{\operatorname{Ric}}\left(W_{2}, \xi\right) & =2 n(\kappa+1) \eta\left(W_{2}\right) \\
\stackrel{\star}{r} & =2 n+r
\end{aligned}
$$

where $\stackrel{\star}{R i}, \stackrel{\star}{R}$ and $\stackrel{\star}{r}$ are the Ricci tensor, the Riemann curvature tensor and scalar curvature admitting the semi-symmetric non-metric connection respectively [24].

The projective curvature tensor $P$ admitting the semi-symmetric non-metric connection is defined by

$$
\stackrel{\star}{P}\left(W_{1}, W_{2}\right) W_{3}=\stackrel{\star}{R}\left(W_{1}, W_{2}\right) W_{3}-\frac{1}{2 n}\left(\stackrel{\star}{\operatorname{Ric}}\left(W_{2}, W_{3}\right) W_{1}-\stackrel{\star}{\operatorname{Ric}}\left(W_{1}, W_{3}\right) W_{2}\right),
$$

for all $W_{1}, W_{2}, W_{3} \in T M$.

\section{Flatness conditions of projective curvature tensor on $(M, \stackrel{\star}{\nabla})$}

In this section, we examine that a $(M, \stackrel{\star}{\nabla})$ is $\xi$-projectively flat, pseudo-quasi-projectively flat and $\phi$-projectively flat.

Definition 3.1. $A(M, \stackrel{\star}{\nabla})$ is called

- $\xi$-projectively flat if we have $\stackrel{\star}{P}\left(W_{1}, W_{2}\right) \xi=0$ for all $W_{1}, W_{2} \in \Gamma(T M)$,

- pseudo-quasi-projectively flat if we have $g\left(\stackrel{\star}{P}\left(\phi W_{1}, W_{2}\right) W_{3}, \phi W_{4}\right)=0$ for all $W_{1}, W_{2}, W_{3} \in \Gamma(T M)$,

- $\phi$-projectively flat if we have $g\left(\stackrel{\star}{P}\left(\phi W_{1}, \phi W_{2}\right) \phi W_{3}, \phi W_{4}\right)=0$ for all $W_{1}, W_{2}, W_{3} \in \Gamma(T M)$.

Theorem 3.2. $A(M, \stackrel{\star}{\nabla})$ is always $\xi$-projectively flat. 
Proof. By putting $W_{3}=\xi$ in (2.10), we obtain

$$
\stackrel{\star}{P}\left(W_{1}, W_{2}\right) \xi=\stackrel{\star}{R}\left(W_{1}, W_{2}\right) \xi-\frac{1}{2 n}\left(\stackrel{\star}{\operatorname{Ric}}\left(W_{2}, \xi\right) W_{1}-\stackrel{\star}{\operatorname{Ric}}\left(W_{1}, \xi\right) W_{2}\right) .
$$

Also from (2.7) and (2.9), we get

$$
\stackrel{\star}{P}\left(W_{1}, W_{2}\right) \xi=R\left(W_{1}, W_{2}\right) \xi-\eta\left(W_{1}\right) W_{2}+\eta\left(W_{2}\right) W_{1}-\frac{1}{2 n}\left(2 n(k+1) \eta\left(W_{2}\right) W_{1}-2 n(k+1) \eta\left(W_{1}\right) W_{2}\right) .
$$

and take into account (2.1), we have

$$
\stackrel{\star}{P}\left(W_{1}, W_{2}\right) \xi=0
$$

for all $W_{1}, W_{2} \in \Gamma(T M)$.

Theorem 3.3. If $(M, \stackrel{\star}{\nabla})$ is pseudo-quasi-projectively flat, then $M$ is an Einstein manifold admitting Levi-Civita connection.

Proof. Using (2.10), we have

$$
g\left(\stackrel{\star}{P}\left(\phi W_{1}, W_{2}\right) W_{3}, \phi W_{4}\right)=\stackrel{\star}{R}\left(\phi W_{1}, W_{2}, W_{3}, \phi W_{4}\right)-\frac{1}{2 n}\left[R i c\left(W_{2}, W_{3}\right) g\left(\phi W_{1}, \phi W_{4}\right)-R^{\star} i c\left(\phi W_{1}, W_{3}\right) g\left(W_{2}, \phi W_{4}\right)\right] .
$$

Let $(M, \stackrel{\star}{\nabla})$ be a pseudo-quasi-projectively flat. Then, by using (2.8) in (3.2), it follows that

$$
\begin{aligned}
\stackrel{\star}{R}\left(\phi W_{1}, W_{2}, W_{3}, \phi W_{4}\right) & =\frac{1}{2 n}\left[\left(\operatorname{Ric}\left(W_{2}, W_{3}\right)-2 n g\left(W_{2}, \phi W_{3}\right)-2 n g\left(h W_{2}, \phi W_{3}\right)+2 n \eta\left(W_{2}\right) \eta\left(W_{3}\right)\right) g\left(\phi W_{1}, \phi W_{4}\right)\right. \\
& \left.-\left(\operatorname{Ric}\left(\phi W_{1}, W_{3}\right)-2 n g\left(\phi W_{1}, \phi W_{3}\right)-2 n g\left(h \phi W_{1}, \phi W_{3}\right)\right) g\left(W_{2}, \phi W_{4}\right)\right]
\end{aligned}
$$

and from (2.6) we get

$$
R\left(\phi W_{1}, W_{2}, W_{3}, \phi W_{4}\right)=\frac{1}{2 n}\left(\operatorname{Ric}\left(W_{2}, W_{3}\right) g\left(\phi W_{1}, \phi W_{4}\right)-\operatorname{Ric}\left(\phi W_{1}, W_{3}\right) g\left(W_{2}, \phi W_{4}\right)\right) .
$$

Take a local orthonormal basis set of $M$ as $\left\{e_{1}, e_{2}, \ldots, e_{2 n}, \xi\right\}$, then $\left\{\phi e_{1}, \phi e_{2}, \ldots, \phi e_{2 n}, \xi\right\}$ is also a local orthonormal basis. Putting $W_{1}=W_{4}=e_{i}$ in (3.3) and summing over $i=1$ to $2 n$, we get

$$
\sum_{i=1}^{2 n} R\left(\phi e_{i}, W_{2}, W_{3}, \phi e_{i}\right)=\frac{1}{2 n}\left[\sum_{i=1}^{2 n}\left(\operatorname{Ric}\left(W_{2}, W_{3}\right) g\left(\phi e_{i}, \phi e_{i}\right)-\operatorname{Ric}\left(\phi e_{i}, W_{3}\right) g\left(W_{2}, \phi e_{i}\right)\right)\right] .
$$

From (2.2) and (2.5), we obtain

$$
\operatorname{Ric}\left(W_{2}, W_{3}\right)=2 n \kappa g\left(W_{2}, W_{3}\right)
$$

Theorem 3.4. Let a $(M, \stackrel{\star}{\nabla})$ be $\phi$-projectively flat. If $\xi$ is Killing vector field, then the manifold is an Einstein manifold.

Proof. Firstly, putting $W_{2}=\phi W_{2}$ and $W_{3}=\phi W_{3}$ in (3.2), we get

$$
g\left(\stackrel{\star}{P}\left(\phi W_{1}, \phi W_{2}\right) \phi W_{3}, \phi W_{4}\right)=\stackrel{\star}{R}\left(\phi W_{1}, \phi W_{2}, \phi W_{3}, \phi W_{4}\right)-\frac{1}{2 n}\left(\stackrel{\star}{\operatorname{Ric}}\left(\phi W_{2}, \phi W_{3}\right) g\left(\phi W_{1}, \phi W_{4}\right)-\stackrel{\star}{\operatorname{Ric}}\left(\phi W_{1}, \phi W_{3}\right) g\left(\phi W_{2}, \phi W_{4}\right)\right) .
$$

Now, by using (2.8) in (3.4) and from definition of $\phi$-projectively flat, it follows that

$$
\begin{aligned}
\stackrel{\star}{R}\left(\phi W_{1}, W_{2}, W_{3}, \phi W_{4}\right) & =\frac{1}{2 n}\left[\left(\operatorname{Ric}\left(\phi W_{2}, \phi W_{3}\right)-2 n g\left(\phi W_{2}, \phi^{2} W_{3}\right)-2 n g\left(h \phi W_{2}, \phi^{2} W_{3}\right)\right) g\left(\phi W_{1}, \phi W_{4}\right)\right. \\
& \left.-\left(\operatorname{Ric}\left(\phi W_{1}, \phi W_{3}\right)-2 n g\left(\phi W_{1}, \phi^{2} W_{3}\right)-2 n g\left(h \phi W_{1}, \phi^{2} W_{3}\right)\right) g\left(\phi W_{2}, \phi W_{4}\right)\right]
\end{aligned}
$$

and from (1.1), we get

$$
R\left(\phi W_{1}, \phi W_{2}, \phi W_{3}, \phi W_{4}\right)=\frac{1}{2 n}\left(\operatorname{Ric}\left(\phi W_{2}, \phi W_{3}\right) g\left(\phi W_{1}, \phi W_{4}\right)-\operatorname{Ric}\left(\phi W_{1}, \phi W_{3}\right) g\left(\phi W_{2}, \phi W_{4}\right)\right) .
$$

For local orthonormal basis $\left\{e_{1}, e_{2}, \ldots, e_{2 n}, \phi e_{1}, \phi e_{2}, \ldots, \phi e_{2 n}, \xi\right\}$ of $M$ by putting $W_{1}=W_{4}=e_{i}$ in (3.5) and summing over $i=1$ to $2 n$, we get

$$
\sum_{i=1}^{2 n} R\left(\phi e_{i}, \phi W_{2}, \phi W_{3}, \phi e_{i}\right)=\frac{1}{2 n}\left[\sum_{i=1}^{2 n}\left(\operatorname{Ric}\left(\phi W_{2}, \phi W_{3}\right) g\left(\phi e_{i}, \phi e_{i}\right)-\operatorname{Ric}\left(\phi e_{i}, \phi W_{3}\right) g\left(\phi W_{2}, \phi e_{i}\right)\right)\right]
$$

From (2.2) and (2.5), we obtain

$$
\operatorname{Ric}\left(\phi W_{2}, \phi W_{3}\right)=2 n \kappa g\left(\phi W_{2}, \phi W_{3}\right)
$$

Also, from (2.4) we have

$$
\operatorname{Ric}\left(W_{2}, W_{3}\right)=2 n \kappa g\left(W_{2}, W_{3}\right)+4(n-1) g\left(h W_{2}, W_{3}\right)
$$

If $\xi$ is Killing vector field then $M$ is an Einstein manifold. 


\section{Symmetry conditions admitting projective curvature tensor on $(M, \stackrel{\star}{\nabla})$}

In this section, we study on a $(M, \stackrel{\star}{\nabla})$ under certain symmetry conditions. We firstly examine $\phi$-projectively semi-symmetric $(M, \stackrel{\star}{\nabla})$ and then we characterize this manifolds satisfying $\stackrel{\star}{Q} . \stackrel{\star}{P}=0$ and $\stackrel{\star}{\text { Ric. }} \stackrel{\star}{P}=0$, where $\stackrel{\star}{Q}$ is the Ricci operator defined by $\stackrel{\star}{\operatorname{kic}}\left(W_{1}, W_{2}\right)=g\left(\stackrel{\star}{Q} W_{1}, W_{2}\right)$.

Definition 4.1. $A(M, \stackrel{\star}{\nabla})$ is said to be $\phi$-projectively semisymmetric if $\stackrel{\star}{P}\left(W_{1}, W_{2}\right) \phi=0$ for all $W_{1}, W_{2} \in \Gamma(M)$.

Theorem 4.2. A $\phi$-projectively $(M, \stackrel{\star}{\nabla})$ is isometric to Example 2.1.

Proof. Suppose $(M, \stackrel{\star}{\nabla})$ be a $\phi$-projectively. Then, we have

$$
\stackrel{\star}{P}\left(W_{1}, W_{2}\right) \phi W_{3}-\phi\left(\stackrel{\star}{P}\left(W_{1}, W_{2}\right) W_{3}\right)=0 .
$$

From (2.10), it follows that

$$
\stackrel{\star}{P}\left(W_{1}, W_{2}\right) \phi W_{3}=\stackrel{\star}{R}\left(W_{1}, W_{2}\right) \phi W_{3}-\frac{1}{2 n}\left(\stackrel{\star}{\operatorname{Ric}}\left(W_{2}, \phi W_{3}\right) W_{1}-\stackrel{\star}{\operatorname{Ric}}\left(W_{1}, \phi W_{3}\right) W_{2}\right) .
$$

Using (2.8) in (4.2), we obtain

$$
\begin{aligned}
\stackrel{\star}{P}\left(W_{1}, W_{2}\right) \phi W_{3} & =\stackrel{\star}{R}\left(W_{1}, W_{2}\right) \phi W_{3}-\frac{1}{2 n}\left\{\operatorname{Ric}\left(W_{2}, \phi W_{3}\right) W_{1}-2 n g\left(W_{2}, \phi^{2} W_{3}\right) W_{1}-2 n g\left(h W_{2}, \phi^{2} W_{3}\right) W_{1}\right\} \\
& +\frac{1}{2 n}\left\{\operatorname{Ric}\left(W_{1}, \phi W_{3}\right) W_{2}-2 n g\left(W_{1}, \phi^{2} W_{3}\right) W_{2}-2 n g\left(h W_{1}, \phi^{2} W_{3}\right) W_{2}\right\} .
\end{aligned}
$$

From (2.1), (2.2) and (2.6), we have

$$
\stackrel{\star}{P}\left(W_{1}, W_{2}\right) \phi W_{3}=\kappa g\left(W_{2}, \phi W_{3}\right) W_{1}-\kappa g\left(W_{1}, \phi W_{3}\right) W_{2}-\frac{1}{2 n} \operatorname{Ric}\left(W_{2}, \phi W_{3}\right) W_{1}+\frac{1}{2 n} \operatorname{Ric}\left(W_{1}, \phi W_{3}\right) W_{2} .
$$

Also, by applying $\phi$ to $\stackrel{\star}{P}$, we get

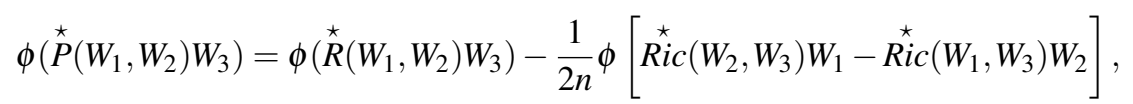

and using (2.8) in (4.4) yields

$$
\begin{aligned}
\phi\left(\stackrel{\star}{P}\left(W_{1}, W_{2}\right) W_{3}\right) & =\phi\left(\stackrel{\star}{R}\left(W_{1}, W_{2}\right) W_{3}\right)-\frac{1}{2 n}\left\{\operatorname{Ric}\left(W_{2}, W_{3}\right)-2 n g\left(W_{2}, \phi W_{3}\right)-2 n g\left(h W_{2}, \phi W_{3}\right)+2 n \eta\left(W_{2}\right) \eta\left(W_{3}\right)\right\} \phi W_{1} \\
& +\frac{1}{2 n}\left\{\operatorname{Ric}\left(W_{1}, W_{3}\right)-2 n g\left(W_{1}, \phi W_{3}\right)-2 n g\left(h W_{1}, \phi W_{3}\right)+2 n \eta\left(W_{1}\right) \eta\left(W_{3}\right)\right\} \phi W_{2}
\end{aligned}
$$

Thus from (2.4) and (2.6), we have

$$
\phi\left(\stackrel{\star}{P}\left(W_{1}, W_{2}\right) W_{3}\right)=\kappa g\left(W_{2}, W_{3}\right) \phi W_{1}-\kappa g\left(W_{1}, W_{3}\right) \phi W_{2}-\frac{1}{2 n} \operatorname{Ric}\left(W_{2}, W_{3}\right) \phi W_{1}+\frac{1}{2 n} \operatorname{Ric}\left(W_{1}, W_{3}\right) \phi W_{2} .
$$

Putting (2.3), (4.3) and (4.5) in (4.1), we have

$$
\begin{aligned}
\stackrel{\star}{P}\left(W_{1}, W_{2}\right) \phi W_{3}-\phi\left(\stackrel{\star}{P}\left(W_{1}, W_{2}\right) W_{3}\right) & =\kappa g\left(W_{2}, \phi W_{3}\right) W_{1}-\kappa g\left(W_{1}, \phi W_{3}\right) W_{2}-\frac{2(n-1)}{2 n}\left[g\left(W_{2}, \phi W_{3}\right)+g\left(h W_{2}, \phi W_{3}\right)\right] W_{1} \\
& +\frac{2(n-1)}{2 n}\left[g\left(W_{1}, \phi W_{3}\right)+g\left(h W_{1}, \phi W_{3}\right)\right] W_{2}-\kappa g\left(W_{2}, W_{3}\right) \phi W_{1}+\kappa g\left(W_{1}, W_{3}\right) \phi W_{2} \\
& +\frac{1}{2 n}\left[2(n-1)\left(g\left(W_{2}, W_{3}\right)+g\left(h W_{2}, W_{3}\right)\right)+2\left(n \kappa-(n-1) \eta\left(W_{2}\right) \eta\left(W_{3}\right)\right)\right] \phi W_{1} \\
& -\frac{1}{2 n}\left[2(n-1)\left(g\left(W_{1}, W_{3}\right)+g\left(h W_{1}, W_{3}\right)\right)+2\left(n \kappa-(n-1) \eta\left(W_{1}\right) \eta\left(W_{3}\right)\right)\right] \phi W_{2}
\end{aligned}
$$

Let take inner product with $W_{4}$ of (4.6) and then to contract $W_{2}$ and $W_{4}$, we obtain

$$
\left\{2 \kappa(1-n)+2\left(\frac{n^{2}-2 n+1}{n}\right)\right\} g\left(W_{1}, \phi W_{3}\right)+\{2(n-1)\} g\left(h W_{1}, \phi W_{3}\right)=0 .
$$


Now, putting $W_{3}=\phi W_{3}$ in (4.7) and from (1.1), we get

$$
\left\{2 \kappa(1-n)+2\left(\frac{n^{2}-2 n+1}{n}\right)\right\} g\left(\phi W_{1}, \phi W_{3}\right)+\{2(n-1)\} g\left(h W_{1}, W_{3}\right)=0 .
$$

Taking trace in both sides of (4.8) and using $t r h=0$, we obtain

$$
\kappa=\frac{n-1}{n} .
$$

Thus $M$ is isometric to Example 2.1.

Theorem 4.3. On a $(M, \stackrel{\star}{\nabla})$, we have $\stackrel{\star}{Q} \cdot \stackrel{\star}{P}=0$.

Proof. For all $W_{1}, W_{2}, W_{3} \in \Gamma(T M)$, we have

$$
\left(\stackrel{\star}{Q}\left(W_{1}\right) . \stackrel{\star}{P}\right)\left(W_{2}, W_{3}\right)=\stackrel{\star}{Q}\left(\stackrel{\star}{P}\left(W_{1}, W_{2}\right) W_{3}\right)-\stackrel{\star}{P}\left(\stackrel{\star}{Q} W_{1}, W_{2}\right) W_{3}-\stackrel{\star}{P}\left(W_{1}, \stackrel{\star}{Q} W_{2}\right) W_{3}-\stackrel{\star}{P}\left(W_{1}, W_{2}\right) \stackrel{\star}{Q} W_{3} .
$$

From (2.8) and (2.9), we have

$$
\stackrel{\star}{Q} W_{2}=2(n-1)\left(W_{2}+h W_{2}\right)+2 n\left(\phi W_{2}+\phi h W_{2}\right)+2(n \kappa+1) \eta\left(W_{2}\right) \xi
$$

and, so

Thus, for $W_{3}=\xi$ in (4.9) we get

$$
\stackrel{\star}{Q} \xi=2 n(\kappa+1) \xi
$$

$$
\left(\stackrel{\star}{Q}\left(W_{1}\right) . \stackrel{\star}{P}\right)\left(W_{2}, \xi\right)=\stackrel{\star}{Q}\left(\stackrel{\star}{P}\left(W_{1}, W_{2}\right) \xi\right)-\stackrel{\star}{P}\left(\stackrel{\star}{Q} W_{1}, W_{2}\right) \xi-\stackrel{\star}{P}\left(W_{1}, \stackrel{\star}{Q} W_{2}\right) \xi-\stackrel{\star}{P}\left(W_{1}, W_{2}\right) \stackrel{\star}{Q} \xi .
$$

From (3.1), (4.10) and (4.11), it follows that

$$
\stackrel{\star}{Q} \cdot \stackrel{\star}{P}=0
$$

Theorem 4.4. $A(M, \stackrel{\star}{\nabla})$ satisfies $\stackrel{\star}{P} \cdot \stackrel{\star}{\text { Ric }}=0$ if and only if $M$ is an Einstein manifold.

Proof. Let $\stackrel{\star}{P} \cdot \stackrel{\star}{R} i c=0$ satisfies on $(M, \stackrel{\star}{\nabla})$, then we get

$$
\stackrel{\star}{\operatorname{Ric}}\left(\stackrel{\star}{P}\left(W_{4}, W_{2}\right) W_{3}, W_{1}\right)+\stackrel{\star}{\operatorname{Ric}}\left(W_{3}, \stackrel{\star}{P}\left(W_{4}, W_{2}\right) W_{1}\right)=0 .
$$

Putting $W_{1}=W_{4}=\xi$ in (4.12), we have

$$
\operatorname{Ric}\left(\stackrel{\star}{P}\left(\xi, W_{2}\right) W_{3}, \xi\right)+\operatorname{Ric}^{\star}\left(W_{3}, \stackrel{\star}{P}\left(\xi, W_{2}\right) \xi\right)=0 .
$$

Also, from (2.10), we get

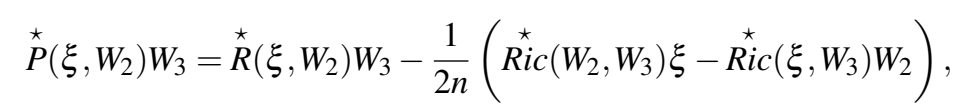

from (2.7), (2.8), (2.9), it follows that

$$
\stackrel{\star}{P}\left(\xi, W_{2}\right) W_{3}=\kappa g\left(W_{2}, W_{3}\right) \xi-\frac{1}{2 n} \operatorname{Ric}\left(W_{2}, W_{3}\right) \xi .
$$

Again putting $W_{3}=\xi$ in (4.14) and using (2.5), we obtain

$$
\stackrel{\star}{P}\left(\xi, W_{2}\right) \xi=0 .
$$

Using (2.9), (4.14) and (4.15) in (4.13), it follows that

$$
\operatorname{Ric}\left(W_{2}, W_{3}\right)=2 n \kappa g\left(W_{2}, W_{3}\right) .
$$

Conversely, let $M$ be an Einstein manifold, i.e $\operatorname{Ric}\left(W_{2}, W_{3}\right)=2 n \kappa g\left(W_{2}, W_{3}\right)$. Then, we get

$$
\stackrel{\star}{P}\left(W_{1}, W_{2}\right) W_{3}=\kappa\left(g\left(W_{2}, W_{3}\right) W_{1}-g\left(W_{1}, W_{3}\right) W_{2}\right)-\frac{1}{2 n}\left(2 n \kappa g\left(W_{2}, W_{3}\right) W_{1}-2 n \kappa g\left(W_{1}, W_{3}\right) W_{2}\right) .
$$

which implies $\stackrel{\star}{P}\left(W_{1}, W_{2}\right) W_{3}=0$. This also give us $\stackrel{\star}{P} \cdot \stackrel{\star}{\star} i c=0$. 


\section{References}

[1] D. E. Blair, Riemannian Geometry of Contact and Symplectic Manifolds, Progress in Mathematics 203, Boston, MA: Birkhauser Boston, Inc., 2002.

[2] D. E. Blair, T. Koufogiorgos, B.J. Papantoniou, Contact metric manifolds satisfying a nullity condition, Israel J. Math., 91 (1995), $189-214$.

[3] S. Tanno, Ricci curvatures of contact Riemannian manifolds, Tohoku Math. J., 40 (1988), 441-448.

[4] D. E. Blair, Two remarks on contact metric structures, Tohoku Math. J., 29 (1977), 319-324.

[5] S. Ghosh, U. C. De, A. Taleshian, Conharmonic curvature tensor on N(k)-contact metric manifolds, ISRN Geometry, (2011), Art. ID 423798, 11 pages.

[6] A. Kazan, S. Kazan Sasakian statistical manifolds with semi-symmetric metric connection, Univers. J. Math. Appl., 1(4) (2018), $226-232$.

[7] M. Y. Y1lmaz, M. Bektaş, Curvature inequalities between a Hessian manifold with constant curvature and its submanifolds, Math. Sci. Appl. E-Notes, 5 (1) (2017), 27-33.

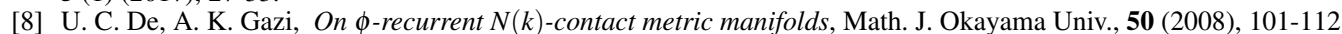

[9] C. Özgur, S. Sular, On N(k)-contact metric manifolds satisfying certain conditions, Sut. J. Math., 44 (2008), 89-99.

[10] N. S. Agashe, M. R. Chafle, A semi-symmetric non-metric connection on a Riemannian Manifold, Indian J. Pure Appl. Math., 23(6) (1992), 399-409.

[11] A. Vanlı Turgut, İ. Ünal, D. Özdemir, Normal complex contact metric manifolds admitting a semi symmetric metric connection, Applied Mathematics and Nonlinear Sciences 5(2) (2020), 49-66.

[12] A. Barman, Semi-symmetric non-metric connection in a P-Sasakian manifold, Novi Sad J. Math., 43 (2013), $117-124$.

[13] A. Barman, U. C. De, Semi-symmetric non-metric connections on Kenmotsu manifolds, Romanian J. Math. Comp. Sci., 5 (2014), 13-24.

[14] U. C. De, S. C. Biswas, On a type of semi-symmetric non-metric connection on a Riemannian manifold, Ganita, 48 (1997), 91-94.

[15] O. C. Andonie, On semi-symmetric non-metric connection on a Riemannian manifold, Ann. Fac. Sci. De Kinshasa, Zaire Sect. Math. Phys., 2 (1976).

[16] P. Majhi, U. C. De, Classifications on N(k)-contact metric manifolds satisfying certain curvature conditions, Acta Math. Univ. Comenianae, 84 (2015), $167-178$.

[17] G. Ayar, S. K.Chaubey, M-projective curvature tensor over cosymplectic manifolds, Differential Geometry-Dynamical Systems, 21 (2019), $23-33$.

[18] G. Ayar, P. Tekin, N. Aktan, Some Curvature Conditions on Nearly Cosymplectic Manifolds, Indian J. Industrial Appl. Math., 10 (2019), 51-60.

[19] G. Ayar and D. Demirhan, Ricci Solitons on Nearly Kenmotsu Manifolds with Semi symmetric Metric Connection, J. Eng. Tech. Appl. Sci., 4(3) (2019), $131-140$.

[20] A. Turgut Vanli, İ.Unal, Conformal, concircular, quasi-conformal and conharmonic flatness on normal complex contact metric manifolds, Int. J. Geom. Methods Mod. Phys., 14(05) (2017), 1750067.

[21] İ. Ünal, R. Sarı, A. Vanlı Turgut, Concircular curvature tensor on generalized kenmotsu manifolds, Gümüşhane Univ. J. Sci. Technol. Inst., (2018), 99-105.

[22] A.Turgut Vanli, İ. Unal, H-curvature tensors on IK-normal complex contact metric manifolds, Int. J. Geom. Methods Mod. Phys., 15(12) (2018) 1850205.

[23] A. Yildiz, U. C. De, M. Cengizhan, K. Arslan, On the Weyl projective curvature tensor of an N(k) -contact metric manifold, Mathematica Pannonica, 21(1) (2010), 1-14.

[24] A. Barman, On $N(k)$-contact metric manifolds admitting a type of a semi-symmetric non-metric connection, Acta Math. Univ. Comenianae, LXXXVI 1 (2017), 81-90

[25] E. Boeckx, A full classification of concat metric ( $k, \mu)$-spaces, Illinois J. Math., 44 (2000), 212-219.

[26] D. E.Blair, J. S. Kim, M. M. Tripathi, On the concircular curvature tensor of a contact metric manifold, J. Korean Math. Soc., 42 (2005), $883-892$. 